\title{
A Review on Methods and Devices for Force Platforms Calibration in Medical Applications
}

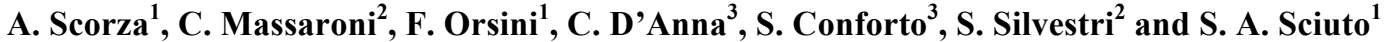 \\ ${ }^{I}$ Department of Engineering, Roma Tre University, via della Vasca Navale 79/81, Roma, Italy \\ ${ }^{2}$ Center for Integrated Research - Unit of Measurements and Biomedical Instrumentation, Università Campus Bio-Medico di Roma, Via \\ Álvaro del Portillo 21 Roma, Italy \\ ${ }^{3}$ Department of Engineering, Roma Tre University, via Volterra 62, Roma, Italy
}

Received 4 December 2017; Accepted 30 December 2017

\begin{abstract}
Nowadays force platforms are widely employed both for diagnostic purposes and for monitoring the execution of motor tasks such as stance, both static and perturbed, and gait. Moreover, even if a clinical assessment based on the knowledge of the forces the patients can exchange with the environment can be useful, some guidelines are needed to determine the practical limitations of the measurements for motion analysis and postural control. In particular, in situ calibration is a fundamental practice especially when the force platforms are either used as reference or are connected with other devices in the measurement chain. Several calibration procedures for force platforms have been proposed in the literature, some of them dealing with only one force component, others with all the force and moment components, and some research groups have also developed and tested some innovative devices or introduced some corrective equations, performing either static or dynamic calibration.
\end{abstract}

Keywords: Calibration, center of pressure, dynamic calibration, force measurement, force platforms

\section{Introduction}

Force platforms (FPs) have a broad range of applications, including crash tests in automobile factories, gait analysis for clinical evaluations, postural assessment and performance analysis in sport motions and gestures [1]. The periodic calibration of these devices guarantees and optimizes the quality of the acquired data. FPs are metal plates with rectangular shape, generally about $0.6 \mathrm{~m} \times 0.6 \mathrm{~m}$ size, with load cells mounted to each corner to have an electrical signal proportional to the load amplitude on the plate. Accurate measurements of the ground reaction force (GRF) with FPs are important in many biomechanics' areas [2], as motion analysis and postural control in both healthy and pathological subjects [3-4]. In a movement analysis laboratory, stereo-photogrammetric motion capture systems and FPs can share one absolute reference frame that allows the computation of joint moments and powers. The correct calibration of the device identifies the geometrical transformation between the FP and the laboratory (generally the absolute one) reference systems, which allows the spatial coherence among the equipment's measurements [5]. Although reliable calibrations of stand-alone stereophotogrammetric systems are today achievable, several errors may affect the FP calibration [6]. Therefore the evaluation of moment and joint forces from gait analysis data strongly depends on the measurement accuracy of the ground reaction force (GRF). Usually, multicomponent FPs are used to measure GRF's components and to calculate the

*E-mail address: andrea.scorza@uniroma3.

ISSN: $1791-2377$ @ 2018. Eastern Macedonia and Thrace Institute of Technology. All rights reserved. doi:10.25103/jestr.111.02 center of pressure (COP) [7] position. It has been shown [8] that the accuracy of the GRFs measured by force plates has a significant impact also on the calculated joint dynamics (to calculate joint forces, moments and powers using inverse dynamics techniques are necessary data collected by force plates and kinematic). Since errors in force plates applications may occur because of improper installation, data acquisition settings $[9,10]$, aging or other damages, an in situ calibration is required to ensure the accuracy of kinetic and dynamic measurements as well as the one of gait analysis results. In order to describe the state-of-the-art this review is divided into three main sections: force platforms description and working principles, force platform calibration (static and dynamic) and clinical use of force platform combined with optoelectronic systems. Force platforms are generally applicable in locomotion studies, both for healthy and pathological patients and in a wide range of technical applications, as shown in table I [11-35]. As in dynamic phenomena such as gait, running, jumping, etc. FPs maybe used in static measurements, e.g. body posture analysis (postural stability and balance).

\section{Force platforms description and working principle}

A force platform is a particular force transducer, where forces and moments applied on a plate are converted into voltage signals. FPs are made by several primary transducers (elastic elements), to convert force components to strain, and secondary transducers, to convert strain into electrical signal: secondary transducers are commonly strain gages or piezoelectric sensors. Commercial devices usually have 3 or 4 load cells placed under the plate (e.g. mounted at each 
corner) where people stand up. In particular FPs can be classified into two main groups:

Table 1. Examples of force platforms applications

\begin{tabular}{|c|c|c|c|c|}
\hline Application & Field & $\begin{array}{c}\text { Measurement } \\
\text { Range }\end{array}$ & $\begin{array}{l}\text { Freq. } \\
\text { range }\end{array}$ & Ref. \\
\hline $\begin{array}{l}\text { Medicine, } \\
\text { Diagnostics, } \\
\text { fall risk, } \\
\text { Biology }\end{array}$ & $\begin{array}{l}\text { Postural } \\
\text { stability } \\
\text { analysis, } \\
\text { locomotion, } \\
\text { Locomotion }\end{array}$ & $0-25000 \mathrm{~N}$ & $0-25 \mathrm{~Hz}$ & [22-26] \\
\hline $\begin{array}{l}\text { Medicine, } \\
\text { Training } \\
\text { assessment, } \\
\text { rehabilitation }\end{array}$ & Medical & $\begin{array}{c}0.5-12.0 \mathrm{~N} \\
0.02-0.70 \mathrm{Nm}\end{array}$ & $0-20 \mathrm{~Hz}$ & [27-29] \\
\hline Free time & Gaming & $0-1500 \mathrm{~N}$ & $0-12 \mathrm{~Hz}$ & {$\left[\begin{array}{ll}30 & -33\end{array}\right]$} \\
\hline $\begin{array}{c}\text { Sport, } \\
\text { Athletics }\end{array}$ & $\begin{array}{c}\text { Performance } \\
\text { evaluation }\end{array}$ & $0-5000 \mathrm{~N}$ & $\begin{array}{c}0-200 \\
\mathrm{~Hz}\end{array}$ & [34-38] \\
\hline $\begin{array}{l}\text { Dynamic } \\
\text { testing of } \\
\text { structures } \\
\text { and } \\
\text { industrial } \\
\text { processes }\end{array}$ & Testing & $\begin{array}{c}0-3.35 \mathrm{kPa} \\
0-10 \mathrm{kN}\end{array}$ & $\begin{array}{c}0-3 \mathrm{~Hz} \\
0-3 \\
\mathrm{kHz}\end{array}$ & $\begin{array}{l}{[39-40]} \\
{[41-42]}\end{array}$ \\
\hline
\end{tabular}

(a) Three component FPs (3FPs), that measure the vertical component of the force applied to the plate (vertical force $\mathrm{F}_{\mathrm{Z}}$ ) as well as the plate moment about the $\mathrm{x}$-axis $\left(\mathrm{M}_{\mathrm{X}}^{\prime}\right)$ and $\mathrm{y}$-axis $\left(\mathrm{M}_{\mathrm{Y}}^{\prime}\right)$, this allows the determination of the two coordinates $\mathrm{x}_{\mathrm{COP}}$ and $\mathrm{y}_{\mathrm{COP}}$ of the force application point (COP, Center of Pressure)

(b) Six component FPs (6FPs), that measure $\mathrm{x}$, y and $\mathrm{z}$ components of the force applied to the plate $\left(\mathrm{F}_{\mathrm{X}}, \mathrm{F}_{\mathrm{Y}}\right.$ , $\mathrm{F}_{\mathrm{Z}}$ ), as well as the plate moment about the $\mathrm{X}$-axis $\left(\mathrm{M}_{\mathrm{X}} \mathrm{X}\right), \mathrm{y}$-axis $\left(\mathrm{M}_{\mathrm{Y}}^{\prime}\right)$ and $\mathrm{z}$-axis $\left(\mathrm{M}_{\mathrm{Z}}\right)$, referred to the origin of the FP reference frame. The output of ${ }^{6} \mathrm{FPs}$ can be equivalently expressed by a measurement of the $\mathrm{x}, \mathrm{y}$ and $\mathrm{z}$ components of the applied force $\left(\mathrm{F}_{\mathrm{X}}\right.$, $\left.\mathrm{F}_{\mathrm{Y}}, \mathrm{F}_{\mathrm{Z}}\right)$, the COP coordinates $\left(\mathrm{x}_{\mathrm{COP}}, \mathrm{y}_{\mathrm{COP}}\right)$ and the vertical torque about the $\mathrm{z}$-axis $\left(\mathrm{T}_{\mathrm{Z}}\right)$ where [43] :

$$
\begin{aligned}
& M^{\prime}{ }_{X}=F_{Z} \cdot y_{C O P} \quad M_{Y}^{\prime}{ }_{Y}=-F_{Z} \cdot x_{C O P} \\
& T_{Z}=-F_{Y} \cdot x_{C O P}+F_{X} \cdot y_{C O P}+M_{Z}
\end{aligned}
$$

6FPs are usually rectangular shaped and have 4 supports (e.g. one for each corner)., where Fx, Fy and Fz are measured (or both the 3 force components and the moment): the six components output of a FP can be obtained by measuring the applied force components for all supports. From the above considerations, the interaction between a body and the FP cannot be completely described by a 3FP, since the horizontal components of the force and the free torque are not measured. Relationships among moments with respect to the FP center and the COP coordinates $\left(\mathrm{x}_{\mathrm{COP}}\right.$, $\left.\mathrm{y}_{\mathrm{COP}}\right)$ are expressed in (2)

$$
\left\{\begin{array}{l}
F_{X}=\sum_{i=1}^{4} f_{X i} \quad F_{Y}=\sum_{i=1}^{4} f_{Y i} \quad F_{Z}=\sum_{i=1}^{4} f_{Z i} \\
M_{X}=b \cdot\left(f_{Z 1}+f_{Z 2}-f_{Z 3}-f_{Z 4}\right) \\
M_{Y}=a \cdot\left(-f_{Z 1}+f_{Z 2}+f_{Z 3}-f_{Z 4}\right) \\
M_{Z}=b \cdot \Delta_{X}+a \cdot \Delta_{Y} \text { where } \Delta_{X}=\left(-f_{X 1}-f_{X 2}+f_{X 3}+f_{X 4}\right) \text { and } \Delta_{Y}=\left(f_{Y 1}+f_{Y 4}-f_{Y 2}-f_{Y 3}\right) \\
x_{C O P}=\frac{-M_{Y}+F_{X} \cdot a_{z 0}}{F_{Z}}=\frac{-M_{Y}^{\prime}}{F_{Z}} \\
y_{C O P}=\frac{M_{X}+F_{y} \cdot a_{z 0}}{F_{Z}}=\frac{M_{X}^{\prime}}{F_{Z}}
\end{array}\right.
$$

where $F_{X}, F_{Y}$ and $F_{Z}$ are the applied force components, $f_{X i}, f_{Y i}$ and $f_{Z i}$ are the components of the force measured by the $\mathrm{i}$-th support. $\mathrm{M}_{\mathrm{X}}$ and $\mathrm{M}_{\mathrm{Y}}$ are the plate moments about the $\mathrm{x}$ and $\mathrm{y}$ axis respectively, $\mathrm{M}_{\mathrm{X}}^{\prime}$ and $\mathrm{M}_{\mathrm{Y}}^{\prime}$ are the plate moments about top plate surface ( $a_{z 0}$ is the top plane offset), $a, b$ and $a_{z 0}$ are distances from the FP axes origin to the origin of the coordinates of the four load cells (figure 1, [43]).

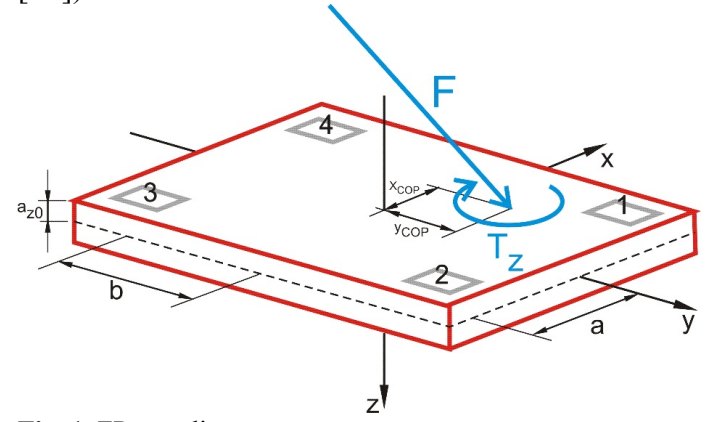

\section{Force Platforms Calibration Approaches}

The calibration process is an operation that, under specified conditions, establishes a relation between the known quantity values with known measurement uncertainties provided by standards and the instrument's indications with associated measurement uncertainties; in a second step, it uses this information to find a relation to obtain a measurement result from an indication [49], this definition can be applied to all biomedical fields [50-60] as the instrument characterization is fundamental to support the diagnosis by means of objective measurements [47-48, 6168]: for FPs a linear model and a linear compensation of the crosstalk between forces, moments and the voltage output are usually assumed as expressed in (3).

Fig. 1. FP coordinate system. 
$\left[\begin{array}{l}F_{X} \\ F_{Y} \\ F_{Z} \\ M^{\prime}{ }_{X} \\ M_{Y}{ }_{Y} \\ M_{Z}\end{array}\right]=\left(\begin{array}{ccc}C_{11} & \cdots & C_{16} \\ \vdots & \ddots & \vdots \\ C_{61} & \cdots & C_{66}\end{array}\right] \cdot\left[\begin{array}{c}V_{1} \\ V_{2} \\ V_{3} \\ V_{4} \\ V_{5} \\ V_{6}\end{array}\right]$

$\left[\begin{array}{l}F_{X} \\ F_{Y} \\ F_{Z} \\ M^{\prime}{ }_{X} \\ M_{Y}{ }_{Y} \\ M_{Z}\end{array}\right]=\mathbf{C}_{\mathbf{i j}}\left[\begin{array}{c}V_{1} \\ V_{2} \\ V_{3} \\ V_{4} \\ V_{5} \\ V_{6}\end{array}\right]+\mathbf{C}_{\mathbf{X i j}}\left[\begin{array}{c}V_{1} \\ V_{2} \\ V_{3} \\ V_{4} \\ V_{5} \\ V_{6}\end{array}\right] M_{X}+\mathbf{C}_{\mathbf{Y i j}}\left[\begin{array}{c}V_{1} \\ V_{2} \\ V_{3} \\ V_{4} \\ V_{5} \\ V_{6}\end{array}\right] M_{Y}$

where $\quad \mathbf{C}_{\mathbf{i j}}=\left(\begin{array}{ccc}C_{11} & \cdots & C_{16} \\ \vdots & \ddots & \vdots \\ C_{61} & \cdots & C_{66}\end{array}\right) \quad \mathbf{C}_{\mathbf{X i j}}=\left(\begin{array}{ccc}C_{X 11} & \cdots & C_{X 16} \\ \vdots & \ddots & \vdots \\ C_{X 61} & \cdots & C_{X 66}\end{array}\right)$

The implementation of (3) or (4) can be obtained by two different approaches, depending on the application of static or dynamic loads: in literature many guidelines for the FPs' calibration, divided into dynamic and static approaches, have been proposed. As some other load cell applications developed in similar fields [69], loading with known weights placed in known positions characterizes the static calibration $[70,71]$. This method is time consuming, estimates the spatial accuracy of the load measurement across the platform and can be affected by load positioning inaccuracies. Some diagnostic modalities, like dynamic posturography [72], require the acquisition of dynamic information and a dynamic calibration to evaluate the FPs response to dynamic loads: testing procedures can involve devices devoted to handle calibrated weights either manually or mechanically. Since the manual handling of masses usually limits the dynamic characterization, the calibration systems using mechanical handling are preferable. In the following, some relevant methods about FPs static and dynamic calibration are explained from literature (Table 2).

\section{A. Static calibration}

Bobbert et al. [73] designed a calibration device in order to apply static vertical forces at more than one hundred calibration points, to compute the measured COP errors, corrected by polynomial regression methods. They proposed a dynamic test where a subject with known mass (i.e. $70 \mathrm{~kg}$ ) ran across a wooden board, equipped in one corner with a stylus placed in a drill hole on an aluminum plate superimposed on the force platform (point loading). The maximal vertical force measured during the tests was about $1700 \mathrm{~N}$. Oscillating signals, related to the frequency of the impact (heel strike) and to the resonance of the force plate $(370 \mathrm{~Hz})$ were acquired using the wooden board and the stylus. In this way, these signals did not contain frequency components related to acquisition chain of the FP. Dynamic calibration was performed using the COP values as reference and neglecting the forces so discarding information dealing with amplitude, direction and frequency of the GRF. Anyway the measurements are affected by some drawbacks: non-linearity, cross-talk among transducers and bending effects of the top plate. To allow safe and quick static testing of the vertical component and COP outputs,
$\mathbf{C}_{\mathrm{Yij}}=\left(\begin{array}{ccc}C_{Y 11} & \cdots & C_{Y 16} \\ \vdots & \ddots & \vdots \\ C_{Y 61} & \cdots & C_{Y 66}\end{array}\right)$

where $\mathrm{C}_{\mathrm{ii}} \quad(\mathrm{i}=1,2, . .6)$ are proportional coefficients between forces (or moments) and voltages, while $\mathrm{C}$ offdiagonal elements describe the crosstalk between variables. In spite of its widespread use, the (3) is not adequate to provide an optimal accuracy when non-linear effects occur, so it should be modified with the non-linear calibration (4):

Gill et al. [74] designed a new load application rig, which enabled with a manually controlled lever system the application of known static vertical forces at several calibration points, reducing the accuracy of measurement and speed of positioning. The equipment used a set of dead weights and provided a vertical load at any point over a rectangle on the floor $\left(1.2 \mathrm{~m}^{2}\right)$. It is made of a rigid frame where two trolleys can be moved orthogonally. The calibration procedure is based on a linear regression between the load mass and the force platform output. In particular a $60 \mathrm{~kg}$ mass is used to apply a maximum vertical load of $1200 \mathrm{~N}$ in any point of the FP surface. From the outputs of the FP (i.e. 6) the COP is determined through the equation provided by the manufacturer. To date, there isn't a method of assessing the accuracy of the GRF horizontal components over the whole FP and it only allows the static performance. Moreover, the parallax error in positioning and the physical size of the rig limit the usefulness of the device, although it has been used to test the accuracy of FPs and reduced dramatically the testing time with respect to other systems. Collins et al. [75] proposed a new method for calibrating FPs to reduce errors related to the CoP location, but also to force and moment components, using an instrumented rod and a least-squares optimization of a linear model of a generic platform system. In particular, the instrumented rod allows for measurement of direction as well as magnitude of forces applied arbitrarily to the force plate, that is accomplished by some optical markers measured by stereophotogrammetric system and a load cell inserted near the tip of the pole: measured quantities may then be transmuted into reference forces and moments vectors applied to the force platform. The use of stereo-photogrammetric system is common in clinical for its stability and reliability [76-77]. The instrumented pole is handled to load each force plate while recording force plate signals and measuring rod load and location. Force plate and load cell signals were acquired at $1200 \mathrm{~Hz}$, while markers were traced at $120 \mathrm{~Hz}$. Loads from 100 to $1000 \mathrm{~N}$ were applied vertically, with simultaneous horizontal loads of $0-250 \mathrm{~N}$ resulting from rod angles of $0-20^{\circ}$ from vertical. Errors could be reduced using as few as 10 locations per standard force platform; however it should be preferable to collect from as many locations as possible, with loads close to real cases. Corrections can 
reduce the effects of misalignment and distortion, and improve the accuracy of force, moment, and COP measurements. It must be pointed out that this method is difficult to apply to simulate dynamic loads in a number and range of locations comparable to the static loads. Moreover the costs and the complexity of the setup are higher than most of the other methods here reported. Rabuffetti et al. [5, 78] in two consecutive works, proposed a method for an optimized platform calibration, using an optoelectronic system, made by an experimental protocol, which measures some mechanical quantities in the platform reference system, and calculates the same quantities in the absolute reference system with a mathematical model. They pointed out how the introduction of such optimized procedure could improve the reliability of the calibrated platform location as well as the kinetic variables in posture and gait analysis. The testing object is a rigid pointed rod, bearing a set of eight reflective markers. Two metal plates, one handheld by an operator and the other placed on the ground, are in contact to both rod extremities and allow to keep the object in equilibrium by means of an operator's compressive force. After the calibration of the optoelectronic system the experimental protocol requires the operator to move the pole, which is pushed against the platform in a conical motion. The experiment is recorded by both the optoelectronic system and the force platform where the platform location and the six-degrees-of-freedom transformation matrix, between the platform and the absolute reference system, are unknown. Is possible to estimate the position of the application point and the GRF direction in the absolute reference frame, without reference to the platform location, using the mathematical model. A possible improvement can be the development of an experimental protocol that is completely independent from human operators. As in [69] the complexity and the costs of the calibration system make it suitable especially for research studies and analysis. In the work of Golberg et al. [79], an optimized calibration is made using the CalTester tool to measure and correct the error existing between the estimated transformation based on a located jig and the measured COP error across a treadmill force plate. To maximize the accuracy of the device in measuring the COP in the motion capture coordinate system, the authors calibrate the device in situ in a manner independent of the motion capture system: data are used to determine the applied moments and the calibration matrix. Errors measured by CalTester are reduced by improving the accuracy of both the COP calculation from force plate data and the COP estimation obtained by using the motion capture data represented in the force plate coordinate system. They highlight a dramatic improvement: the proposed method reduces the COP error to $1.0 \mathrm{~mm}$ and the angle error to $0.8^{\circ}$ for medial/lateral orientation and of about $1.6 \mathrm{~mm}$ and $0.6^{\circ}$ for the anterior/posterior position. Their procedure increases the accuracy of instrumented treadmills after installation by a static calibration, introducing some correction concepts that can be applicable to other treadmill models. In some published studies [80-82], carried on by the same research group, a theoretical and experimental validation of a portable system for the in-situ calibration of six-component FPs is presented. The system is made up of a device, a data-acquisition procedure and an algorithm. A least-squares algorithm, improved by a previous one [80], that estimates the FP re-calibration matrix, was designed and tested using a simulation approach. The algorithm is the core of this portable system and it assumes the calibration inputs known, 3-D, time-varying loads, applied to the FP at known coordinates, and not forced in their direction of application [81]. Applying simple sinusoidal loads in five different points, the algorithm can guarantee errors lower than $0.2 \mathrm{~N}$ and $0.4 \mathrm{Nm}$ when calculating force and moment components of an applied load. The device, that is simple and lightweight, is equipped with a high-precision 3-D load cell. The data-acquisition procedure lasts about $1 \mathrm{~h}$ and requires up to 13 measurements consisting of manual positioning the load cell on the force platform, and then having the operator applying loads on both load cell and force platform by body motion. The procedure provides loads in the same range of posture and gait tests. The system was validated on four commercial force platforms: the residual errors in the computation of the center of pressure (i.e. the distance between the measured and actual COPs) were $2.3 \pm 1.4 \mathrm{~mm}$, $2.6 \pm 1.5 \mathrm{~mm}, 11.8 \pm 4.3 \mathrm{~mm}, 14.0 \pm 2.5 \mathrm{~mm}$ before recalibration, $1.1 \pm 0.6 \mathrm{~mm}, 1.8 \pm 1.1 \mathrm{~mm}, 1.0 \pm 0.6 \mathrm{~mm}, 3.2 \pm 1.1$ $\mathrm{mm}$ after global re-calibration, and $0.7 \pm 0.4 \mathrm{~mm}, 0.8 \pm 0.5$ $\mathrm{mm}, 0.5 \pm 0.3 \mathrm{~mm}, 2.0 \pm 1.2 \mathrm{~mm}$ after local re-calibration. The force platform re-calibration influenced the sign, value, and timing of net joint moments, calculated during a gait task through an inverse dynamics approach. Anyway, the dynamic range and repeatability of the calibration system are limited by the human operator, as the load is provided manually.

In addition calibrated force platforms could be used as gold standard systems, for calibration of force/torque sensors [83]. In this calibration method, the force/torque sensor is rigidly connected to a force platform and during a dynamic functional loading procedure force/torque data are synchronously recorded. In this way an accurate calibration matrix for the force/torque sensor can easily be obtained via least-squares optimization. Seven different loading methods were compared and the calibration matrices were evaluated based on the unprocessed data from the seven loading methods individually and all loading methods combined. The calibration matrices' performance was subsequently compared in an in situ trial where five common work tasks (e.g., manual lifting, pushing, walking) were performed by an operator while standing on the force platform wearing a "Force Shoe" sensorized with two calibrated force/torque sensors attached to its sole. Root-mean-square differences (RMSDs) between the force/torque sensor and force platform measures were evaluated over all tasks. The use of the calibration matrices based on all loading combined methods resulted in small RMSDs (center of pressure: $<2$ mm, GRF: $<8 \mathrm{~N}$ ).

Recently some cheap force platforms are commercial available, thanks to the increasing demand lead to technological innovations in the gaming market. Many biomechanics studies allow the integration of this kind of device, in different research areas [84, 85]. A work group [85] has introduced the Wii Balance Board (WBB), as a device useful to quantify the center of pressure. They examined its validity in comparison with the 'gold standard' consisting of a laboratory-grade force platform. Thirty healthy subjects performed, with eyes closed or open on two separate occasion, a combination of single and double leg standing balance tests. Both devices exhibited good to excellent COP path length test-retest reliability withindevice (Intraclass Correlation Coefficient, ICC $=0.66-0.94$ ) and between-device (ICC $=0.77-0.89$ ) on all testing protocols. Examination of the Bland Altman Plot discovered no relationship between the mean and the difference in any test, however in three of the four tests the minimum 
detectable change (MDC) values for the WBB did exceed those of the force platform. These findings suggest that the WBB is a valid tool for evaluating standing balance. In conclusion, the WBB provides comparable data to a force platform when assessing COP path length during standing balance trials. Consequently, the WBB has the potential to fill the gap between clinical assessment of standing balance and laboratory testing, a theory confirmed also in another latest study [86]. In a recent study [87] static calibration on a low-cost force platform (WBB) is performed in order to determine its force and COP accuracy and reliability for assessment of postural control, performing an uncertainty analysis, the authors used WBBs to provide future users information about the repeatability and accuracy of the WBB force and COP data. Across WBBs, it has been found the total uncertainty of force measurements to be within 9.1 $\mathrm{N}$, and of COP location within $4.1 \mathrm{~mm}$, which is much higher than the recommended uncertainty of $0.1 \mathrm{~mm}$ for posturographic applications. However, repeatability of a single measurement within a board was better $(4.5 \mathrm{~N}, 1.5$ $\mathrm{mm}$ ), suggesting that the WBB is the best to be used for relative measurements using the same device, instead of absolute measurement across devices. Internally recorded calibration values were comparable to those evaluated experimentally. In combination with a prior evaluation of WBB performance and published standards for measuring human balance, this study provides necessary information to evaluate the use of the WBB for analysis of human balance control. In conclusion the WBB use has been suggested for onlylow-resolution measurements and low-frequency movements, because it cannot be considered equivalent to laboratory-grade force plates.

\section{B. Dynamic calibration}

As shown above many approaches have been performed to provide static calibration of force platforms while dynamic calibration methods are not well established yet. Recently, the requirements for measuring dynamic forces have become more severe and varied in many industrial and research applications [88-95] so increasing the importance of the procedures for dynamic calibration of FPs [96]. Cappello et al. [80] described a new technique based on a least-squares approach. An accurate estimation of a force platform calibration matrix has been proposed: this is based on simple manual procedures and is particularly useful when the applied loads directions cannot be perfectly aligned with the axes of the FP. This new procedure allows the combined application of vertical and horizontal forces, both static and time-varying to all force platforms. To reduce the bias in the estimated calibration matrix parameters, the robust calibration method includes the angular errors in the least-squares parameter vector, thus. The performance of the robust method were compared with the ones obtained by conventional approaches, using a numerical simulation approach starting from a known calibration matrix. Fujii et al. [96] proposed a new method for dynamic calibration, in which an impulse is given to the FP by a moving mass and the load value is evaluated through an optical interferometer as a change in momentum of the mass. In the experimental set-up a pneumatic linear bearing is used to give an impulse to a force transducer. The maximum weight of the moving part is approximately $270 \mathrm{~N}$ and the impulse (i.e. the time integration of the impact force) which is detected by the semiconductor strain gauge force transducer, is acquired at 1 $\mathrm{kHz}$ sampling frequency. The relative uncertainty in the measurement of the impulse acting on a force transducer of this method is estimated to be less than $10^{-3}$. Even if the method is limited by arbitrary settings of amplitude and frequency of the dynamic force, it has some advantages in the measurement accuracy of the impulse amplitude on the transducer and in the transducer arrangement. Fairburn et al. developed a pendulum system [97], which could be mounted to a FP to allow completion of two testing stages: (1) assessment of the visual vector and temporal GRF system and (2) comparison between the measured forces and a theoretical profile (in-depth analysis of force and angular displacement). The visual vector system has been implemented to help in the alignment of orthotic bracing and prosthetic limbs: a real-time display of the GRF is provided in the stance phase and overlaid on a video image of the subject loading the FP. Aiming at comparing the force values, a box-section framework was designed and built to be bolted to the force platform: for visual testing a $20 \mathrm{~kg}$ spherical lead pendulum swung freely within the boundaries of the mounting frame and the evaluation of the force platform performance was attempted comparing the theoretical pendulum force profile with the measured force data collected during pendulum oscillations. Errors into the measurement results are related to mechanical design of the system and to manual handling. In addition, the current pendulum mass is limited to $20 \mathrm{~kg}$, that is a drawback for the very narrow frequency range of force solicitations as well for its amplitude, that is not adequate to adult gait measurements. Anyway the calibration system could be transportable, that is advantageous over many other devices here reported. In a recent study Hong-Jung Hsieh et al. [98] developed a device for both static and dynamic calibration and an Artificial Neural Network (ANN) based correction method [99]. The calibration device is based on the principle of leverage to control the amplitudes and positions of the loads applied to the FP under test: it is made of a base secured to the floor by eight industrial pads, a loading rod that moves along an arm that rotates and moves along an axis relative to the base, and a plate that carries standard weights and moves along the arm. The calibration system provides a grid of 121 calibration points, and applies vertical loads of $650 \mathrm{~N}, 800 \mathrm{~N}$ and $100 \mathrm{~N}$ at each point, where forces, moments and COP were measured at a sampling rate of $120 \mathrm{~Hz}$ in $2 \mathrm{~s}$ (static calibration). The uncertainty of the calibration load was less than $0.007 \mathrm{~N}$ and errors in the COP and GRF are at minimum around the center of the FP. The dynamic calibration is conducted at the center of the FP, by moving a $200 \mathrm{~N}$ weight on a holder backward and forward. At higher dynamic loads the calibration of the COP position is conducted asking a subject with a body mass of $60 \mathrm{~kg}$ to stand with one leg on the holder, and with the other leg on a platform with the same height placed outside the FP: the dynamic condition during walking could be simulated by shifting from two-legs stance to single-stance. This calibration is performed to simulate 3 vertical loading ranges: $800-1400 \mathrm{~N}, 650-800 \mathrm{~N}$ and 450-650 N. Forces and moments are collected at a sampling rate of $1000 \mathrm{~Hz}$. An ANN trained with static calibration data seems to be effective in correcting errors. This new device together with the ANN method is useful for accurate COP and GRF measurements in human motion analysis. Anyway errors in COP depend on the loading velocity and although they could be (partially) corrected by the ANN algorithms, further studies are needed to investigate how inclusion of dynamic calibration data in the training of the ANN could improve the error correction.

The aim of the work of N. P. Linthorne [100] is to show 
how a FP analysis of the standing vertical jump may be used in the dynamics and kinematics analysis of one-dimensional motion. In it the curves of displacement and velocity of the jumper's center of mass are plotted by means of a numerical integration of the force-time records from a FP. The above curves allow to study the relationships among the resulting acceleration, displacement, and velocity of the body and the forces acting on a body: in particular the curves of forcetime, displacement-time, velocity-time, force-displacement, and acceleration-time are calculated from the GRF (measured through the FP). Three methods to calculate the height of the jump (impulse-momentum method, workenergy method and flight time method,) were described and compared giving rise to the conclusion that the work-energy method is very sensitive to the correct selection of the instant before the jump where the jumper is stationary and the GRF is equal to the jumper's body weight. A research group [101] have designed, and validated a portable force platform (PFP) for recording instantaneous vertical GRFs in vertical jumps. The platform was constructed of 2 steel plates each $70 \mathrm{~cm} \times 70 \mathrm{~cm}$ and was calibrated with 3 sets of known weights in 9 positions on the PFP. Data were collected by 25 raw samples per region and per weight to assure that regions of the PFP do not respond differently: the only statistically significant difference was the main effect for weight. PFP calibration was performed by correlation between 17 known weights and voltage outputs, showing a linear correspondence. The PFP was placed on top of an AMTI force platform and vertical jumps were performed while sampling from both platforms simultaneously at 500 $\mathrm{Hz}$. The PFP natural frequency was approximately $100 \mathrm{~Hz}$. The PFP appears to be an accurate, simple, and cheap way to measure vertical component force in vertical jumps. The work of Luciani et al. [102] introduces a new mechatronic device (SENLY) that provides balance perturbations while subjects carry out daily motor tasks (e.g., walking, upright stance). SENLY perturbs belts movements in the horizontal plane using two independently-controlled treadmills that destabilize balance. It is also equipped with force sensors, which can be used to estimate the GRFs and identify events along the gait cycle in order to trigger the platform perturbation. In this work the calibration matrix was estimated by the technique developed by Cappello et al. [8082]. Conversely, dynamic approaches reported in literature either adopt quite slow (e.g. 1-2 Hz) movements respect to the platform frequency range, or their weights are too light therefore introducing potential inaccuracies. For these evaluations, a calibration protocol was defined and applied to set up the calibration matrix of each sensed platform: it consisted of a comparison between COP and GRF components evaluated by SENLY related to a three-axial load cell placed between force plates and an external walkway, by means of a removable reference grid. The reference grid was a $2100 \mathrm{~mm} \times 1050 \mathrm{~mm}$ sheet of aluminum, which was clamped to the sensed surface. For each of the two plates, the data acquisition procedure accounted for records in 9 known positions on the entire surface. A $10 \mathrm{~s}$ long record was acquired with $1000 \mathrm{~Hz}$ sample rate for each position. The algorithm minimizes the Root Mean Square (RMS) of the residual error by estimating 36 parameters. The movement of the subject on the walkway during calibration procedures generated variable loads, which components on vertical and horizontal directions, ranged between $500 \mathrm{~N}$ and $1000 \mathrm{~N}$, and $-50 \mathrm{~N}$ and $150 \mathrm{~N}$ respectively, while the COP deviated of about $15 \mathrm{~mm}$ from the center of the load cell. As expected, calibration improved both accuracy and precision of the measurements, decreasing RMS (of about 50\%) and maximum error, increasing correlation coefficients between estimated and applied variables. Moreover, it allowed achieving a better estimation of measurements than those adopted for other FPs of similar size, meeting all required specifications, with a negligible influence of the instrumental noise. Another approach to apply dynamic load for calibrating FPs is the use of parallel robots, because their accuracy, load capacity and velocity range make them suitable to simulate dynamic patterns as those that occur in biomechanical studies [103]. In [104] a method for FP recalibration by means of a 3DOF parallel robot instrumented with a calibrated load cell is proposed: the device provides patterns of forces within a wide range of forces and frequencies [105], similar to that in walking, running or any other gesture in biomechanical studies [103]. The forces are applied on the platform to be calibrated through a calibrated load cell (that is assumed to be the gold standard): a calibration grid is used to place the sensor at different locations on the platform through a steel ball that was located on the cell to provide a point contact. Two types of load patterns were applied in the study: a) actual gait patterns and b) calibration load patterns, i.e. a 0.5 $\mathrm{Hz}$ sinusoidal load of null minimum value and $500 \mathrm{~N}$ peak to peak. In the calibration procedure the load cell is placed at $\mathrm{m}$ different locations and for each of them $\mathrm{n}$ load forces are applied: if ${ }^{j} \mathrm{~F}_{\mathrm{Ci}}$ is the $\mathrm{i}$-th force $(\mathrm{i}=1,2, \mathrm{n})$ measured by the load cell at the $\mathrm{j}$-th location $(\mathrm{j}=1,2, . . \mathrm{m})$ and ${ }^{\mathrm{j}} \mathrm{F}_{\mathrm{i}}$ corresponds to the same force measured by the FP, their difference ${ }^{j} \mathrm{dF}_{\mathrm{i}}={ }^{\mathrm{j}} \mathrm{F}_{\mathrm{Ci}}{ }^{\mathrm{j}} \mathrm{F}_{\mathrm{i}}$ in (5) is due to two main sources of error: the former is related to errors in the sensitivity coefficients, the other is the alignment of load cell and FP local reference systems.

$$
{ }^{j} d \mathbf{F}_{i}={ }^{j} \mathbf{F}_{i}-{ }^{j} \mathbf{F}_{C i}=\sum{ }^{j} d f_{H k} \cdot \mathbf{u}_{H k}+{ }^{j} d f_{V k} \cdot \mathbf{u}_{V k}
$$

Where $\mathbf{u}_{\mathrm{Hk}}$ and $\mathbf{u}_{\mathrm{Vk}}$ are the unit vectors corresponding to the horizontal $(\mathrm{H})$ and the vertical $(\mathrm{V})$ direction respectively associated with sensor $\mathrm{k}$, and ${ }^{j} d f_{H k}$ and ${ }^{j} d f_{V k}$ come from differentiating the relationship (6) between the horizontal $f_{H}$ and vertical $f_{V}$ force components and the corresponding output voltage $\mathrm{V}_{\mathrm{Hk}}$ and $\mathrm{V}_{\mathrm{Vk}}$ measured by the horizontal and vertical channels of the FP:

$$
\left[\begin{array}{c}
V_{H k} \\
V_{V k}
\end{array}\right]=\left[\begin{array}{cc}
S_{d H} & S_{c H} \\
S_{c V}+S_{m V} & S_{d V}
\end{array}\right]_{k}\left[\begin{array}{c}
f_{H k} \\
f_{V k}
\end{array}\right]=[\mathbf{s}]_{k}\left[\begin{array}{c}
f_{H k} \\
f_{V k}
\end{array}\right]
$$

Where $S_{\mathrm{dH}}$ and $\mathrm{S}_{\mathrm{dV}}$ are the direct sensitivity coefficients, $\mathrm{S}_{\mathrm{cH}}$ and $\mathrm{S}_{\mathrm{cV}}$ are the cross-talk sensitivity coefficients, $\mathrm{S}_{\mathrm{mV}}$ is the nonlinear cross-sensitivity coefficient, that models the nonlinear cross-talk effect associated to deformation of the transducer when a horizontal force is applied. By applying (5) to the $n$ measurements at the $m$ locations of the load cell, a system with $3 \times n \times m$ equations and $5 \times \mathrm{k}$ variables that can be solved by least squares. On the other hand the misalignment error is modeled as a small rotation, ${ }^{\mathrm{j}} \mathrm{d} \theta$ that propagates in the forces measured by the load cell, i.e. ${ }^{j} \mathrm{~d} \mathbf{F}_{\mathrm{Ci}}=$ ${ }^{j} \mathrm{~d} \boldsymbol{\theta} \times{ }^{\mathrm{j}} \mathbf{F}_{\mathrm{Ci}}$. The calculation of ${ }^{\mathrm{j}} \mathrm{d} \boldsymbol{\theta}$ can be obtained from [106] and the calibration process consists of an iterative process in which the Eqs. (5) and ${ }^{j} \mathrm{~d} \boldsymbol{\theta}$ are consecutively solved until it converges into a stationary solution of the sensitivity coefficients. With the aim to overcome the drawbacks due to system transportability in a recent work [107] the authors designed and realized a steel framework equipped with 4 
wheels, for the transport in situ application, and a mechanic actuator with helicoidally gear driven by an electric motor. At the head of the actuator is fixed a load cell that lean on the FP during the calibration procedure. The system allows to load the plate at any point of its surface with different inclinations to calibrate FP's COP and GRF. The proposed system is still under characterization.

\section{Conclusion}

Because of their great versatility, diagnostic relevance and easiness of use, force platforms are today widespread in different diagnostic fields. To perform an accurate analysis some specifications that are mainly related to motion bandwidth, maximum payload of the plate and force components are required. In particular, an accurate calibration is required for both static and dynamic conditions, nevertheless methods reported in the scientific literature are numerous, diversified, not standardized and often more effective for static than dynamic conditions, ranging from the use of calibrated masses to the more complex parallel robots. Moreover calibration approaches can be based on linear or non linear models that require algorithms of different complexities. Anyway the dynamic calibration of force platforms still seems to be a topic where further investigations are needed, from the definition of standard protocols for dynamic performance assessment of the force platforms to the precise and repeatable generation of dynamical load patterns similar to that in human gait and posture. Finally, in recent years a lot of clinical applications has used FPs with optoelectronic systems to integrate dynamics with kinematic features, in order to reduce the risk of misunderstanding and misinterpreting data. In spite of promising results and diagnostic capabilities, further questions rise about the calibration of the whole integrated system but seem to be not widely discussed in scientific and technical literature yet.

\section{Conflict of Interest}

The authors declare that they have no conflict of interest.

\section{Ethical Standard}

This article does not contain any studies with human participants or animals performed by any of the authors.

This is an Open Access article distributed under the terms of the Creative Commons Attribution License

\section{References}

1. C. D'Anna, M. Schmid, A. Scorza, S. A. Sciuto, L. Lopez, S. Conforto, Open Biomed Eng J., 11: 49-58, (2017)

2. R. Cross, American Journal of Physics, 1998, 67(4), 304-309.

3. F. Orsini, S. Scena, C. D'Anna, A.Scorza,L. Schinaia, S.A. Sciuto, $22^{\text {nd }}$ IMEKO TC4 International Symposium \& 20th International Workshop on ADC Modelling and Testing Supporting World Development Through Electrical \& Electronic Measurements, Iasi, Romania, September 14-15, (2017)

4. D. Bibbo, I. Bernabucci, A. Scorza, F. Orsini, S.A. Sciuto, $22^{\text {nd }}$ IMEKO TC4 International Symposium \& 20th International Workshop on ADC Modelling and Testing Supporting World Development Through Electrical \& Electronic Measurements, Iasi, Romania, September 14-15, (2017)

5. M. Rabuffetti, M. Ferrarin, F.Benvenuti. Med. Bio. Eng. Comput. , 39, 638-643, (2001).

6. Y. Ehara, H. Fujimoto, S. Miyazaki, M. Mochimaru, S. Tanaka, S. Yamamoto, Gait and Posture, 5, 251-255, (1997)

7. J. Middelton, P. Sinclair, R. Patton, Clinical Biomechanics, 14, 357-360, (1999)

8. S.T. McCaw, P. DeVita, J. Biomech, 28, 985-8, (1995)

9. M. Schmid, S. Conforto, V. Camomilla, A. Cappozzo, T. D'Alessio, Medical Engineering and Physics, 24(9):623-31, (2002)

10. S. A. Sciuto, A. Scorza, 4th European Conference of the International Federation for Medical and Biological Engineering, IFMBE Proceedings, Vol. 22, pp. 110-113, Antwerp, (2009)

11. M. H. de Groot, J. P. C. M. van Campen, M. A. Moek, L. R. Tulner, J. H. Beijnen, C. J. C. Lamoth, Drugs \& Aging, Volume 30, Issue 11, pp 901-920, (2013)

12. Thomas Maribo, Berit Schiøttz-Christensen, Lone Donbæk Jensen, Niels Trolle Andersen, Kristian Stengaard-Pedersen, Eur Spine J , 21:425-431, (2012)

13. Bhattacharya, R. Morgan, R. Shukla, H.K. Ramakrishanan, L. Wang, Annals of Biomedical Engineering, Vol. 15, pp. 533-550, (1987)

14. G. Bizzo, N. Guillet, A. Patat, P. M. Gagey, Med \& Biol Eng \& Comput, 23, 474-476, (1985)

15. F. Chorin, A. Rahmani, B. Beaune, C. Cornu, Aging Clin Exp Res ,27:473-482, (2015)

16. R. Kram, T. M. Griffin, J. M. Donelan and Y. H. Chang, Journal of Applied Physiology Published ,Vol. 85 no. 2, 764-769, (1998)

17. M. Schmid, S. Conforto, L. Lopez, T. D'Alessio, Experimental Brain Research, 179(3):375-385, (2007)
18. M. Schmid, S. Conforto, L. Lopez, P. Renzi, T. D’Alessio, Journal of NeuroEngineering and Rehabilitation, 2(29), (2005)

19. S. Conforto, M. Schmid, V. Camomilla, T. D'Alessio, A. Cappozzo, Gait \& Posture, 14(1):28-35, (2001)

20. C. D'Anna, D. Bibbo, M. Goffredo, M. Schmid, S. Conforto, IFMBE Proceedings, 41:1755-1758, (2014)

21. C. D'Anna, D. Bibbo, C. De Marchis, M. Goffredo, M. Schmid, S. Conforto, IEEE-EMBS International Conference on Biomedical and Health Informatics, BHI 2014, 6864382, pp. 380-383, (2014)

22. Biewener, R. Blickhan, A. K. Perry, N. C. Heglund, C. R. Taylor, Journal of Experimental Biology 137: 191-205, (1988)

23. T. Farley, J. Glasheen, T. A. McMahon, Journal of Experimental Biology 185: 71-86, (1993)

24. Ann C. Zumwalta, Mark Hamrickb, Daniel Schmitta, Journal of Biomechanics Volume 39, Issue 15, Pages 2877-2881, (2006)

25. R. J. Full, M. S. Tu, Journal of Experimental Biology 148: 129146,(1990)

26. R. M. Alexander, Journal of Experimental Biology 115: 231-238, (1985)

27. Tim Horeman, Sharon P. Rodrigues, Frank-Willem Jansen, Jenny Dankelman, John J. van den Dobbelsteen, Surg Endosc 24:31023108, (2010)

28. Abhishek Srivastava, Arun B. Taly, Anupam Gupta, Senthil Kumar, Thyloth Murali, Journal of the Neurological Sciences 287 89-93, (2009)

29. Pasquale Arpaia, Pasquale Cimmino, Ernesto De Matteis, Gianni D’Addio, Measurement 51 , 400-410, (2014)

30. Dae-Sung Park, GyuChang Lee, Journal of NeuroEngineering and Rehabilitation, 11:99, (2014)

31. Julia M. Leach,Martina Mancini, Robert J. Peterka, Tamara L. Hayes and Fay B. Horak, Sensors, 14, 18244-18267, (2014)

32. Daniel J Goble, Brian L Cone and Brett W Fling, Journal of NeuroEngineering and Rehabilitation, 11:12, (2014)

33. Ross A. Clark,Adam L. Bryant, Yonghao Pua, Paul McCrory, Kim Bennell, Michael Hunt, Gait \& Posture 31 , 307-310, (2010)

34. Poul Dyhre-Poulsen, Eur J Appl Physiol, 56:390-397, (1987)

35. M. Izquierdo, X. Aguado, R. Gonzalez, L. Lopez, K. Hakkinen , Eur J Appl Physiol , 79: 260 - 267, (1999)

36. N. P. Linthorne, Am. J. Phys. 69 (11), (2001)

37. E. L.M. Naves, A. A. Pereira, A. O. Andrade, A. B. Soares, Measurement 42, 449-455, 2009

38. F. Crenna, G. B. Rossi, L. Bovio, 19th IMEKO World Congress 2009, Volume 2, Pages 943-948, Lisbon, Portugal, (2009).

39. J. L. Sanborn, Purdue University Lafayette, Indiana, (1965) 


\section{A.Scorza, C. Massaroni, F. Orsini, C. D'Anna, S. Conforto, S. Silvestri and S. A. Sciuto/}

Journal of Engineering Science and Technology Review 11 (1) (2018) 10-18

40. A. Ebrahimpour, R. L. Sack, ASCE, Journal of Structural Engineering, 118 (4) pp. 1121-1136, (1992)

41. S.Z. Meymand, M. Ahmadian, Measurement 81, 113-122, (2016)

42. G. Totis, O. Adams , M. Sortino, D. Veselovac, F. Klocke, Measurement 49, 164-181, (2014)

43. Kistler force plate formulae. 2008. [Online]. Available at: http://isbweb.org/software/movanal/vaughan/kistler.pdf. Accessed on: November 10, (2017)

44. P. Cappa, S. Silvestri, S. A. Sciuto, Measurement Science and Technology, 12 (9), 1439-1444, (2001)

45. A. Proto, D. Bibbo, S. Conforto, M. Schmid, IEEE 2014 Biomedical Circuits and Systems Conference, BioCAS 2014 Proceedings 6981722, pp. 300-303, (2014)

46. F.P. Branca, P. Cappa, S.A. Sciuto, S. Silvestri, Journal of Clinical Engineering, 22 (3), pp. 163-170, (1997)

47. F. Orsini, A. Scorza, A. Rossi, F. Botta, S.A. Sciuto, R. di Giminiani, 2016 IEEE International Symposium on Medical Measurements and Applications (MEMEA 2016) Proceedings, Benevento, Italy, pp.137-142, (2016)

48. A. Rossi, F. Orsini, A. Scorza, F. Botta, S.A. Sciuto, R. di Giminiani, 2016 IEEE International Symposium on Medical Measurements and Applications (MEMEA 2016) Proceedings, Benevento, Italy, pp.131-136, (2016)

49. International vocabulary of metrology - Basic and general concepts and associated terms (VIM), JCGM 200:2012,(2012)

50. M. Savic, G. Geršak, Measurement 59, 192-197, (2015)

51. L. Battista, A. Scorza, S.A. Sciuto, Lecture Notes in Electrical Engineering, 268 LNEE, pp. 453-457, (2014)

52. Ki Woo Kim, Applied Microscopy, 46(2): 71-75, (2016)

53. F. Marinozzi, F. Bini, A. D'Orazio, A. Scorza, (IST 2008) Chania, Island of Crete, Greece. p. 50-55, (2008)

54. V. Ramamohan, J. T. Abbott, G. G. Klee, Y. Yih, Measurement 50 $175-185,(2014)$

55. F. Marinozzi, F.P. Branca, F. Bini, A. Scorza, Measurement: Journal of the International Measurement Confederation, 45 (5), pp. 1334-1342, (2012)

56. A. Belkova, D. R Pichora, R. E Ellis, Int J CARS, 11:521-527, (2016)

57. T. Liu, Y. Inoue, K. Shibata, Measurement 42, 978-988, (2009)

58. L. Battista , S.A. Sciuto, A. Scorza, Proceedings of the Ninth IASTED International Conference on Biomedical Engineering (BioMed 2012). Innsbruck, Austria, February 15 - 17, p. $443-$ 449,(2012)

59. R. Di Marco, S. Rossi, E. Castelli, F. Patanè, C. Mazzà, P. Cappa, Measurement, Volume 101, Pages 265-271, (2017)

60. L. Battista, A. Scorza, G. Lupi, S.A. Sciuto, 20th IMEKO TC4 International Symposium, Benevento, Italy, (2014)

61. A. Rossi, F. Orsini, F. Botta, A. Scorza, L. Schinaia, D. Bibbo, S.A. Sciuto, 22nd IMEKO TC4 International Symposium \& 20th International Workshop on ADC Modelling and Testing Supporting World Development Through Electrical \& Electronic Measurements, Iasi, Romania, (2017)

62. F. Orsini, A. Rossi, A. Scorza, F. Botta, S.A. Sciuto, 22nd IMEKO TC4 International Symposium \& 20th International Workshop on ADC Modelling and Testing Supporting World Development Through Electrical \& Electronic Measurements, Iasi, Romania, (2017)

63. P. Castellini, L. Scalise, G.M. Revel, Measurement 27 , 29-42, (2000)

64. J. Ogorevc, G. Geršak, D. Novak, J. Drnovšek, Measurement 46, 2993-3001, (2013)

65. L. Schinaia, A. Scorza, F. Orsini, S. A. Sciuto, 22nd IMEKO TC4 International Symposium \& 20th International Workshop on ADC Modelling and Testing Supporting World Development Through Electrical \& Electronic Measurements, Iasi, Romania, (2017)

66. F. Lamonaca, M. Vasile b, A. Nastro, Measurement 92 , 89-95, (2016)

67. L. Schinaia, A. Scorza, F. Orsini, S. A. Sciuto, 22nd IMEKO TC4 International Symposium \& 20th International Workshop on ADC Modelling and Testing Supporting World Development Through Electrical \& Electronic Measurements, Iasi, Romania, (2017)

68. P. Arpaia, F. Clemente, C. Romanucci, Measurement , 41, 10401044, (2008)

69. S. Conforto, S.A. Sciuto, D. Bibbo, A. Scorza, ECIFMBE 2008, IFMBE Proceedings (22): 106-109, (2008)

70. N. Chockalingam, G. Giakas, A. Iossifidou, Gait Posture, 16, 233237, (2002)

71. M. G. Hall, H. E. Fleming, M. J. Dolan, S. F. Millbank, J. P. Paul, Biomech, 29,659-665, (1996)
72. C. De Marchis, F. Patané, M. Petrarca, S. Carniel, M. Schmid, S. Conforto, E. Castelli, P. Cappa, T. D'Alessio, IFMBE Proceedings, 41, pp. 69-72, (2014).

73. M. F. Bobbert, H. C. Schamhardt, J. Biomech., 23, 705-710, (1990)

74. H. S. Gill, J. J. O'Connor, Gait and posture,5,228-232, (1997)

75. S. H. Collins, P. G. Adamczyk, D. P. Ferris, A. D. Kuo, Gait and posture, 29, 59-64, (2009)

76. C. D’Anna, S. Scena, A. Scorza, M. Schmid, F. Orsini, S. A Sciuto, S. Conforto, 2017 IEEE International Instrumentation and Measurement Technology Conference (I2MTC 2017) Proceedings, Torino, Italy, pp. 962-966, (2017).

77. C. Massaroni, E. Schena, F. Bastianini, A. Scorza, P. Saccomandi, G. Lupi, F. Botta, S.A. Sciuto, S. Silvestri, the Open Biomedical Engineering Journal, 8, pp. 120-130, (2015)

78. M. Rabuffetti, M. Ferrarin, P. Mazzoleni, F.Benvenuti, A. Pedotti, Gait and Posture, 17, 75-80, (2003)

79. S. R. Goldberg, T. M. Kepple, S. J. Stanhope, Journal of applied biomechanics, 25, 401-4, (2009)

80. A. Cappello, D. Lenzi, L. Chiari, Med. Biol. Eng. Comput., 42, 350-5, (2004)

81. A. Cedraro, A. Cappello, L. Chiari, Gait \& Posture, 28, 488-494, (2008)

82. A. Cedraro, A. Cappello, L. Chiari, Gait \& Posture, 29, 449-453, (2009)

83. G. S. Faber, C. Chang, I. Kingma, H. M. Schepers, S. Herber, P. H.Veltink, J. T. Dennerlein, Journal of Biomechanics, 45, 1332 1338, (2012)

84. W. Young, S. Ferguson, S. Brault, C. Craig, Gait \& Posture, (2010).

85. R. A. Clark, A. L. Bryant, Y. Pua, P. McCrory, K. Bennell, M. Hunt, Gait \& Posture, 31, 307-310, (2010)

86. D. P. Fransz, I. Kingma, J. H. van Dieen. Journal of biomechanics, 46 (7), 1392-1395, (2013)

87. H. L. Bartlett, L. H. Ting, J. T. Bingham, Gait \& Posture, 39, 224 228,2014

88. A. Scorza, F. Orsini, S.A. Sciuto, I2MTC 2017 - 2017 IEEE International Instrumentation and Measurement Technology Conference, Proceedings, art. no. 7969844, (2017)

89. A. Rossi, F. Orsini, A. Scorza, F. Botta, F. Leccese, E. Silva, K. Torokhtii, I. Bernabucci, S.A. Sciuto, 22nd IMEKO TC4 International Symposium \& 20th International Workshop on ADC Modelling and Testing Supporting World Development Through Electrical \& Electronic Measurements, Iasi, Romania, (2017)

90. W. G. Weekers, P. H. J. Schellekens, Measurement Vol. 20, No. 3, pp. 197-209, (1997)

91. P. Baron, M. Koc iško, L. Blaško, P. Szentivanyi, Measurement 96, 24-33, (2017)

92. A. Scorza, D. Pietrobon, F. Orsini, S. A. Sciuto, 22nd IMEKO TC4 International Symposium \& 20th International Workshop on ADC Modelling and Testing Supporting World Development Through Electrical \& Electronic Measurements, Iasi, Romania, (2017)

93. H. M. Hsu, Y. C. Lin, W. Lin, C. J. Lin, Y. L. Chao, L. C. Kuo, Measurement 46, 506-513, (2013)

94. A. Panjan, M. Supej, J. Rosker, N. Sarabon, Measurement 85, 13 19, (2016)

95. R. Araneo, A. Rinaldi, A. Notargiacomo, F. Bini, M. Pea, S. Celozzi, F. Marinozzi and G. Lovat. Design Concepts, Fabrication and Advanced Characterization Methods of Innovative Piezoelectric Sensors Based on ZnO Nanowires. Sensors-Basel $14: 12,23539-23562,(2014)$

96. Y. Fujii, H. Fujimoto. Meas. Sci. Technol., 10, 31-33, (1999)

97. P.S. Fairburn, R. Palmer, J. Whybrow, S. Fielden, S. Jones, Gait and Posture, 12, 25-33, (2000)

98. H. J. Hsieh, T.W. Lu, S.C. Chen, C. M. Chang, C. Hung, Gait \& Posture, 33, 701-7, (2011)

99. P. Daponte, D. Grimaldi, Measurement 23, 93 - 115, (1998)

100.N. P. Linthorne. American Journal of Physics, 69 (11), 1198-1204, (2001)

101.J. A. Major, W. A. Sands, J. R. McNeal, D. D. Paine, and R. Kipp, Journal of Strength and Conditioning Research, 12 (1), 37-41, (1998)

102.L. B. Luciani, V. Genovese, V. Monaco, L. Odetti, E. Cattin, S Micera, Journal of Neuro Engineering and Rehabilitation, (2012).

103.M. Díaz-Rodríguez, V. Mata, V. Valera, A. Page, Mechanisms and Machine Theory, 45(9), pp 1337-1356, (2010)

104.E. Brau, J. Cazalilla, M. Vallés, A. Besa, A. Valera, V. Mata and A. Page, Proceedings of the International Conference on Biomedical Electronics and Devices (BIODEVICES-2013), pages 132-136, (2013) 
A.Scorza, C. Massaroni, F. Orsini, C. D'Anna, S. Conforto, S. Silvestri and S. A. Sciuto/

Journal of Engineering Science and Technology Review 11 (1) (2018) 10-18

105.M. Vallés, M. Díaz-Rodríguez, A. Valera,V. Mata, A. Page, MUSME 2011, the Int. Symposium on Multibody Systems and Mechatronics,2011, Valencia, Spain, (2011).

106.A. Page, H. de Rosario, V. Mata, C. Atienza, Journal of Mechanical Design 131, 031005 (8 pp.), (2009)
107.F. Orsini, A. Rossi, A. Scorza, S.A. Sciuto, 2017 IEEE International Instrumentation and Measurement Technology Conference (I2MTC 2017) Proceedings, Torino, Italy, pp. 10821087, (2017) 